Research Paper

\title{
LncRNA-H19 regulates chemoresistance to carboplatin in epithelial ovarian cancer through microRNA-29b-3p and STAT3
}

\author{
Xueye Tian ${ }^{1 *}$, Xiaohang Zuo ${ }^{2 *}$, Meng Hou ${ }^{1}$, Chen Li $^{1}$, Yue Teng1 ${ }^{\circledR}$ \\ 1. Department of Obstetrics and Gynaecology, The First Affiliated Hospital of Xi' an Jiaotong University, Xi' an 710061, China \\ 2. Xijing Hospital, Fourth Military Medical University, Xi'an 710032, China \\ * These authors have contributed equally to this work. \\ $\square$ Corresponding author: Yue Teng (Corresponding author), Ph.D., M.D., Department of Obstetrics and Gynecology, the First Affiliated Hospital of Xi'an \\ Jiaotong University, Xi'an 710061, China. E-mail: tengyue@xjtufh.edu.cn; Fax: (86)029 8525 2580; Tel: (86) 02985323836
}

(c) The author(s). This is an open access article distributed under the terms of the Creative Commons Attribution License (https://creativecommons.org/licenses/by/4.0/). See http://ivyspring.com/terms for full terms and conditions.

Received: 2021.02.03; Accepted: 2021.07.19; Published: 2021.07.25

\begin{abstract}
Background: Platinum-based chemotherapy is part of current standard treatment for epithelial ovarian cancer (EOC). However, chemoresistance often rapidly developed, leading to chemotherapy failure and unfavored prognosis. Increasing evidence has demonstrated the important role of oncogenic long noncoding RNA H19 in various cancers, including EOC. No current study is available in exploring the role of IncRNA-H1 9 in carboplatin resistance of EOC and its underlying mechanism.

Methods: Levels of IncRNA-H19, miR-29b-3p, and STAT3 mRNA were measured by qRT-PCR. The $50 \%$ inhibitory concentration value was detected with Cell Counting Kit-8 (CCK8). Colony-formation and CCK8 assays were employed to measure cell viability. Cell migration and invasion ability was evaluated with transwells. Western blot assay was utilized to measure P-gP, MRPI, LRP, and STAT3 protein levels. The targeting between IncRNA-H19 and miR-29b-3p, as well as miR-29b-3p and STAT3, was verified by dual-luciferase, RNA immunoprecipitation, and RNA pull-down experiments.

Results: IncRNA-H19 and STAT3 were sharply increased, while miR-29b-3p was decreased in carboplatin-resistant EOC. Carboplatin efficacy was enhanced by IncRNA-H19 silencing in chemo-resistant EOC cells. IncRNA-H19 served as a competing endogenous RNA of miR-29b-3p, causing the derepression of miR-29b-3p downstream target STAT3, leading to chemoresistance in carboplatin-tolerated EOC.

Conclusions: The IncRNA-H19/miR-29b-3p axis improved carboplatin resistance of EOC by targeting STAT3, indicating a possible approach to improving chemotherapy for EOC.
\end{abstract}

Key words: IncRNA-H19, miR-29b-3p, STAT3, carboplatin resistance, epithelial ovarian cancer

\section{Introduction}

Epithelial ovarian cancer (EOC) is the most malignant and lethal gynecological disease and is characterized by the early onset of disseminated metastasis. Cytoreduction and platinum-based chemotherapy constitute the current standard treatment for EOC, which is effective when treatment begins. However, as treatment continues, most EOC patients become resistant to chemotherapy [1]. Exploration of the underlying molecular mechanism of chemoresistance is urgently needed for effective treatment for EOC.

Noncoding RNAs refer to a large class of RNA transcripts with no ability to encode proteins that have recently emerged as promising regulatory molecules in various biological and pathological processes, including X-chromosome deactivation, stem cell revival, cellular differentiation, apoptosis and invasion [2] [3] [4]. According to their lengths, 
regulatory ncRNAs are categorized into small ncRNAs $(<200 \mathrm{nt})$ and long noncoding RNAs (lncRNAs; $\geq 200 \mathrm{nt}$ ) [5]. Intriguingly, different types of ncRNAs are found to interact with each other to exert their functions. For instance, lncRNAs have been found to regulate microRNAs (miRNAs) by serving as competing endogenous RNAs, and miRNAs are often found to regulate gene expression by binding to mRNAs at their $3^{\prime}$ untranslated regions (UTRs) [6]. Among a variety of identified lncRNAs, lncRNA-H19 has been found to be differentially expressed and participate in the onset and development of ovarian cancer [7] [8]. Some studies have shown that the level of lncRNA-H19 is abnormally elevated in ovarian cancer tissues, and H19 may be involved in the cisplatin resistance of EOC cells [9]. To date, there is no study available on the role of lncRNA-H19 in EOC carboplatin resistance. In our earlier studies, we explored the biological functions of miR-29b-3p in EOC development and found that miR-29b-3p is an important regulator of energy metabolism, EMT, and epigenetic mechanisms of EOC [10] [11] [12]. miR-29b-3p was also identified as a target of lncRNA-H19 in recent studies [9]. Considering the involvement of lncRNA-H19 and miR-29b-3p in EOC progression, we aimed to determine whether the interaction between lncRNA-H19 and miR-29b-3p contributes to the regulation of chemoresistance in EOC. Notably, using bioinformatics software, we previously identified signal transducer and activator of transcription 3 (STAT3) as a predicted miR-29b target, suggesting that miR-29b-3p participates in regulation of chemoresistance via the STAT3 pathway. Currently, no research has explored the role of the IncRNA-H19-miR-29b-3p-STAT3 axis in EOC chemoresistance. Elucidating the role of the lncRNA-H19-miR-29b-3p-STAT3 axis in EOC chemoresistance will help to better understand EOC and provide novel clinical therapies for EOC.

\section{Materials and Methods}

\section{Cell lines and human tissue specimens}

The human ovarian cancer cell line SKOV3 (obtained from ATCC, Manassas, VA, USA) and the carboplatin-resistant human ovarian cancer cell lines SKOV3-CB (obtained from Cancer Research Institute of Guangxi Medical University) were maintained in high-glucose DMEM (Gibco, Invitrogen, Carlsbad, CA, USA) supplemented with $10 \%(\mathrm{v} / \mathrm{v})$ fetal bovine serum (Gibco, Invitrogen, Carlsbad, CA, USA) at $37^{\circ} \mathrm{C}$ in a humidified $5 \% \mathrm{CO}_{2}$ atmosphere [13]. SKOV3-CB cells were preserved in a final concentration of 5 $\mathrm{nmol} / \mathrm{L}$ carboplatin to maintain their resistance. This study was approved by the Ethics Committee of The
First Affiliated Hospital of Xi' an Jiaotong University, China (study reference No.: XJTU1AF2018LSK-245). Written consent was obtained from each study participant enrolled. twenty high-grade serous ovarian cancer samples and twenty normal ovarian tissue samples were used in our study. These samples were all newly diagnosed, previously untreated, and collected from patients at The First Affiliated Hospital of Xi'an Jiaotong University, PR China between 2013 and 2014. Detailed diagnostic and pathological reports were collected for all patients, and none of them had been previously treated with chemotherapy.

\section{Bioinformatics analysis}

Potential targets and binding sites of miR-29b-3p were predicted using several online programs, including miRanda (http://www.microrna.org/ microrna/home.do) and TargetScan (http://www .targetscan.org/); STAT3 was predicted to be a downstream target gene of miR-29b-3p by both software programs, and this finding was further validated by experiments.

\section{Plasmids and cell transfection}

Short interfering RNA (siRNA) for lncRNA-H19 (si-H19; $50 \mathrm{nM}$ ), negative control siRNA (si-NC; 50 nM), lncRNA-H19 overexpression plasmid (pcDNA3.1-H19; $2 \mu \mathrm{g}$ ), pcDNA3.1 $(2 \mu \mathrm{g})$, miR-29b-3p mimic $(60 \mathrm{nM})$, miR-29b-3p inhibitor $(120 \mathrm{nM})$, STAT3-overexpression plasmid (pcDNA3.1-STAT3; 2 $\mu \mathrm{g}$ ), STAT3 siRNA (si-STAT3, $2 \mu \mathrm{g}$ ) and negative control (NC) were all purchased from RiboBio (Guangzhou, China). Ovarian cancer cells were seeded into 6-well plates until 50\%-60\% confluency and transiently transfected with targeted siRNAs using X-treme GENE siRNA Transfection Reagent (Roche, Indianapolis, IN, USA) according to the manufacturer's instructions. Forty-eight hours after transfection, the cells were collected for further experiments.

\section{Quantitative real-time PCR}

PCR experiments were performed as indicated in our former studies [10]. The primers for IncRNA-H19, miR-29b-3p and $u 6$ reverse transcription and amplification were designed by and purchased from RiboBio Co., Ltd. (Guangzhou, China). The primer sequences were as follows: STAT3: F: GTCAGGCACC ATTTTAGGCAC, R: TCAGGCTTTCCTTGACCAGT; lncRNA-H19: F: TACAACCACTGCACTACCTG, R: TGGAATGCTTGAAGGCTGCT; miR-29b-3p: F: ACACTCCAGCTGGGTAGCACCATTTGAAATC, R: TGGTGTCGTGGAGTCG; u6: F: CTCGCTTCGGC AGCACA; R: TGGTGTCGTGGAGTCG. 


\section{Viability assay and IC50 determination}

A total of $5 \times 10^{3}$ cells were seeded in 96-well plates and treated with increasing concentrations of carboplatin (from $1000 \mu \mathrm{M}$ to $15.625 \mu \mathrm{M}$ by 2-fold dilution steps) or a combination of carboplatin (from $1000 \mu \mathrm{M}$ to $15.625 \mu \mathrm{M}$ by 2 -fold dilution steps) and H19 $(50 \mu \mathrm{M}) 24 \mathrm{~h}$ after seeding. At $48 \mathrm{~h}$ or $72 \mathrm{~h}$ after treatment, cell viability was determined by a colorimetric assay using a tetrazolium salt, WST-1 (Roche). A nonlinear regression was performed, and the relative IC50 values were determined with GraphPad Prism.

\section{Colony-forming assay}

Transfected SKOV3-CB cells were seeded into 6-well plates at a density of 500 cells per well. Following incubation at $37^{\circ} \mathrm{C}$ and $5 \% \mathrm{CO}_{2}$ for 2 weeks, visible colonies were fixed and stained with $0.1 \%$ crystal violet (Sigma-Aldrich Co.), and the number of colonies was counted under an inverted microscope (Olympus, Tokyo, Japan).

\section{Cell migration and invasion assay}

In vitro cell migration assays were performed using Transwell chambers ( $8 \mu \mathrm{M}$ pore size; Costar). After reaching $75 \%-80 \%$ confluency, cells were serum-starved overnight. After being detached with trypsin and washed with PBS, the cells were then resuspended in serum-free medium, and $500 \mu$ cell suspensions $\left(5 \times 10^{5}\right.$ cells $\left./ \mathrm{ml}\right)$ were added to the upper chamber. Complete medium was added to the bottom chambers. The cells that had not migrated were removed from the upper face of the filters using cotton swabs, while the migrated cells were fixed with $5 \%$ glutaraldehyde solution and stained with $0.5 \%$ solution of toluidine blue in $2 \%$ sodium carbonate. Images of five random $\times 10$ fields were captured from each membrane, and the number of migratory cells was counted, with the mean value of five duplicate assays for each experimental condition used for statistical analysis. Similar inserts coated with Matrigel were used to determine invasive potential in the invasion assay.

\section{Western blot analysis}

WB experiments were performed as indicated in our former studies [10]. Mouse anti-human STAT3 (ab119352, Abcam, Cambridge, UK), rabbit anti-human P-gp (ab235954, Abcam, Cambridge, UK), mouse anti-human MRP1 (ab24102, Abcam, Cambridge, UK), rabbit anti-human LRP (ab92544, Abcam, Cambridge, UK), and mouse anti-human $\beta$-actin (\#3700S, CST, MA, USA) were incubated with the membranes overnight at $4^{\circ} \mathrm{C}$ at dilutions of $1: 800$, 1:800, 1:800, 1:800, and 1:2000, respectively.

\section{Luciferase reporter assay}

The putative binding regions of lncRNA-H19 and miR-29b-3p or miR-29b-3p and STAT3 were predicted by bioinformatics analysis [14]. Briefly, it is predicted by starBase software that lncRNA-H19 could target miR-29b-3p. miRanda and TargetScan software predicted STAT3 as a downstream target of miR-29b-3p. Luciferase reporter assay was employed to confirm the binding of the genes. Partial sequences of lncRNA-H19 containing wild-type or mutant binding sites of miR-29b-3p were cloned into the psiCHECK ${ }^{\mathrm{TM}}-2$ plasmid (Promega Corporation, Fitchberg, WI, USA) to generate the wild-type plasmid (H19-WT) or mutant-type plasmid (H19-MUT). Similarly, wild-type or mutant plasmid STAT3 3'UTR-WT or STAT3 3'UTR-MUT was also constructed using the same approach. The luciferase reporter plasmid was transfected into SKOV3-CB cells together with miR-NC or miR-29b-3p. Relative firefly luciferase activity, normalized with Renilla luciferase, was then measured $48 \mathrm{~h}$ after transfection with a dual-luciferase reporter gene assay system (Promega, Madison, WI, USA), and the results are shown as the percentage change over the appropriate control. Since complementary binding between lncRNAs, miRNAs and mRNAs leads to decreased expression of the downstream gene, a decreased luciferase activity indicates the binding of specific gene sequences inside reporter plasmids.

\section{RNA immunoprecipitation (RIP)}

RIP analysis was conducted in SKOV3-CB cells using the Magna RIP RNA-Binding Protein Immunoprecipitation Kit (EMD Millipore) according to the manufacturer's instructions. In brief, SKOV3-CB cells were transfected with miR-29b-3p or miR-NC for $48 \mathrm{~h}$. Then, the cells were lysed in RIP buffer, and cell extraction was incubated with Protein A/G magnetic beads bound with primary antibodies against Ago2 (Abcam) or IgG (Abcam). The protein and DNA in the immunoprecipitated complex were removed, and the enrichment levels of lncRNA-H19 were measured by qRT-PCR.

\section{RNA pull-down}

The interaction between lncRNA-H19 and miR-29b-3p was examined using the Pierce Magnetic RNA-Protein Pull-Down Kit (Thermo Fisher Scientific) according to the manufacturer's protocols. Biotin-labeled wild-type lncRNA-H19 (Bio-H19 WT) containing the putative miR-29b-3p binding sites and the biotin-labeled mutant lncRNA-H19 (Bio-H19 MUT) designed to disrupt the base pairing between lncRNA-H19 and miR-29b-3p were mixed with protein extracts of SKOV3-CB cells and M-280 
streptavidin-coated magnetic beads (Invitrogen). The beads were subsequently washed with ice-cold lysis buffer, low-salt buffer, and high-salt buffer. Next, bound RNAs were purified from the RNA-protein complex, and miR-29b-3p expression was determined by qRT-PCR.

\section{Statistical analysis}

Each experiment was independently performed at least 3 times. Data are presented as the mean \pm standard deviation (SD) and were analyzed using GraphPad Prism software. Statistical significance was assessed using two-tailed unpaired Student's t-test. When a $P$ value was less than 0.05 , the differences were considered statistically significant.

\section{Results}

\section{IncRNA-H19 expression characteristics in EOC and its correlation with the prognosis of EOC patients}

20 primary EOC tissues and 20 normal ovarian tissues were collected and analyzed for lncRNA-H19 levels. The qRT-PCR results indicated that the lncRNA-H19 levels were higher in the EOC tissues than those in the normal ovarian tissues (Fig. 1A). We then employed Kaplan-Meier analysis to evaluate the correlation between lncRNA-H19 levels and EOC patient survival. The EOC patients were categorized into high lncRNA-H19 expression group (lncRNAH19 expression > median) and low lncRNA-H19 expression group (lncRNA-H19 expression $\leq$ median). EOC patients with high lncRNA-H19 levels showed poor survival (Fig. 1B). These results indicated lncRNA-H19's involvement in EOC development.

\section{LncRNA-H19 strengthened ovarian cancer cell resistance to carboplatin}

We then tested the cytotoxic effects of carboplatin on two matched human ovarian cancer cell lines: SKOV3 (wild type), and SKOV3-CB (SKOV3 after long-term carboplatin treatment). Increasing concentrations of carboplatin were given, and cell numbers after $48 \mathrm{~h}$ were measured by colorimetric assays. A carboplatin concentration-dependent decrease in viability was observed in both EOC cells (Fig. 2A-B). The relative IC50 of carboplatin was measured at $48 \mathrm{~h}$ and $72 \mathrm{~h}$ after incubation. As shown in Fig. 2C-D, SKOV3-CB cells had a higher IC50 value than SKOV3 cells. To test whether lncRNA-H19 regulates EOC cell sensitivity to carboplatin, we treated EOC cells with different concentrations of carboplatin with $50 \mu \mathrm{M}$ of si-lncRNA-H19. Intriguingly, si-lncRNA-H19 substantially lowered the viability of carboplatin-resistant cells incubated with carboplatin with a decrease in the IC50 (Fig. 2C-D). Thus, lncRNA-H19 potently strengthened the chemoresistance of carboplatin-resistant EOC cells. lncRNA-H19 knockdown in response to specific lncRNA-H19 siRNAs is shown in Fig. S1. Two lncRNA-H19 siRNAs were used in preliminary experiments, and siRNA 1 was chosen and used in subsequent experiments.

\section{LncRNA-H19 and miR-29b-3p levels between EOC and control tissues/cells}

We tested the lncRNA-H19 and miR-29b-3p levels in the tissues and cells of EOC patients and healthy controls. Table 1 shows the pathological features of the EOC patients included in the study. As shown in Fig 3A, lncRNA-H19 expression was much higher in the EOC tissues than that in the normal ovarian tissues $(\mathrm{P}<0.05)$, and miR-29b-3p expression in the EOC tissues was much lower than that in the normal ovarian tissues $(\mathrm{P}<0.05)$. Furthermore, IncRNA-H19 expression in SKOV3-CB cells was higher than that in SKOV3 cells $(\mathrm{P}<0.05)$; however,

B

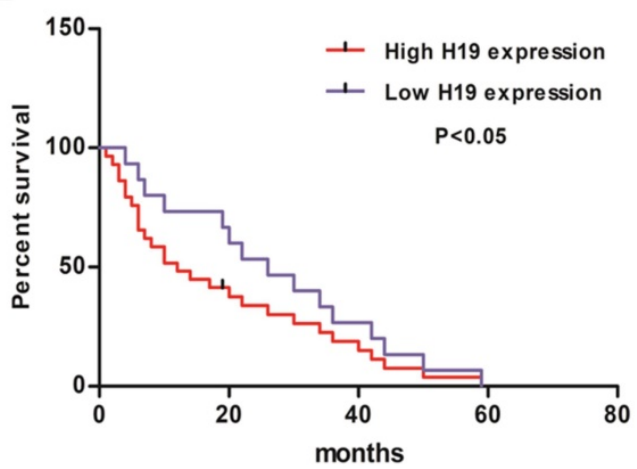

Figure 1: IncRNA H19 expression characteristics in EOC and its correlation with EOC prognosis. A) IncRNA-H19 levels were detected by qRT-PCR in 20 primary EOC tissues and normal ovarian tissues. Data are shown as the mean \pm SEM. B) IncRNA-H19 was considered high when the expression of IncRNA-H 19 was greater than the average, and IncRNA-H19 was regarded as low when the expression of IncRNA-H19 was lower than the average. The Kaplan-Meier curve showing the overall survival of EOC patients with high/low IncRNA-H19 levels was established. 
miR-29b-3p was significantly lower in SKOV3-CB cells was higher than that in SKOV3 cells $(\mathrm{P}<0.05)$ (Fig. 3B). To explore the miR-29b-3p/lncRNA-H19 correlation, we measured the expression levels of miR-29b-3p and lncRNA-H19 in the sera of 20 patients. A negative correlation was found between the two ncRNAs ( $\mathrm{r}=-0.704$; Fig. 3C).

Table 1. Correlation between $\mathrm{H} 19$ expression and clinicopathologic characteristics of EOC patients.

\begin{tabular}{llll}
\hline Clinicopathologic Parameters & \multicolumn{2}{l}{ H19 expression } & \multirow{2}{*}{$P$} \\
\cline { 2 - 3 } & High & Low & 0.3291 \\
\hline Age (years) & 8 & 6 & \\
55 and above & 2 & 4 & 0.0246 \\
Under 55 & 3 & 8 & \\
FIGO stage & 7 & 2 & 0.0062 \\
I-II & & & \\
III-IV & 7 & 1 & 0.1775 \\
Differentiation & 3 & 9 & \\
Well and moderate & & 7 & \\
Poorly & 4 & & \\
Lymph node metastasis & Absent &
\end{tabular}
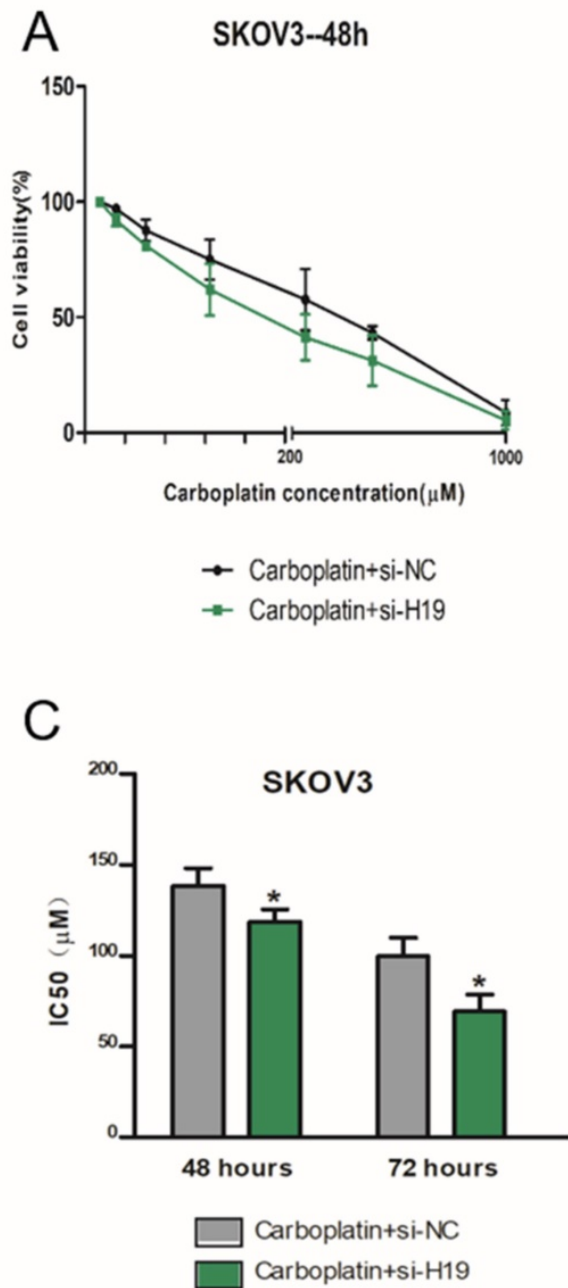

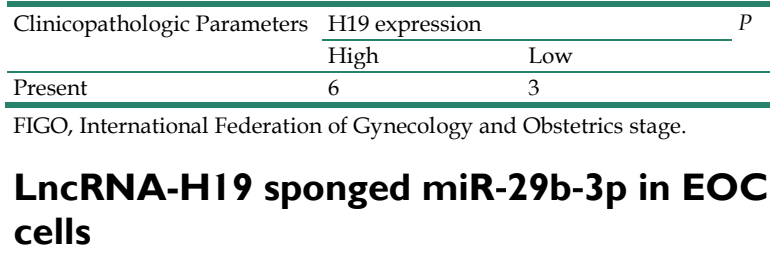

A major mechanism of lncRNAs is to competitively bind to its target miRNAs, which buffers miRNAs' inhibitory effect on their downstream targets. The negative correlation between lncRNA-H19 and miR-29b-3p suggested a potential negative regulatory mechanism between the two ncRNAs. Starbase 2.0 was employed to predict targeting sites for lncRNA-H19 and miR-29b-3p (Fig. 4A). A dual-luciferase reporter gene assay showed that compared with the cells transfected with pmirGLO-H19-Wt and miR-NC, the cells transfected with pmirGLO-H19-Wt and miR-29b-3p mimics showed a strong decrease in luciferase activity $(\mathrm{P}<0.05)$ (Fig. 4B). No significant change in luciferase
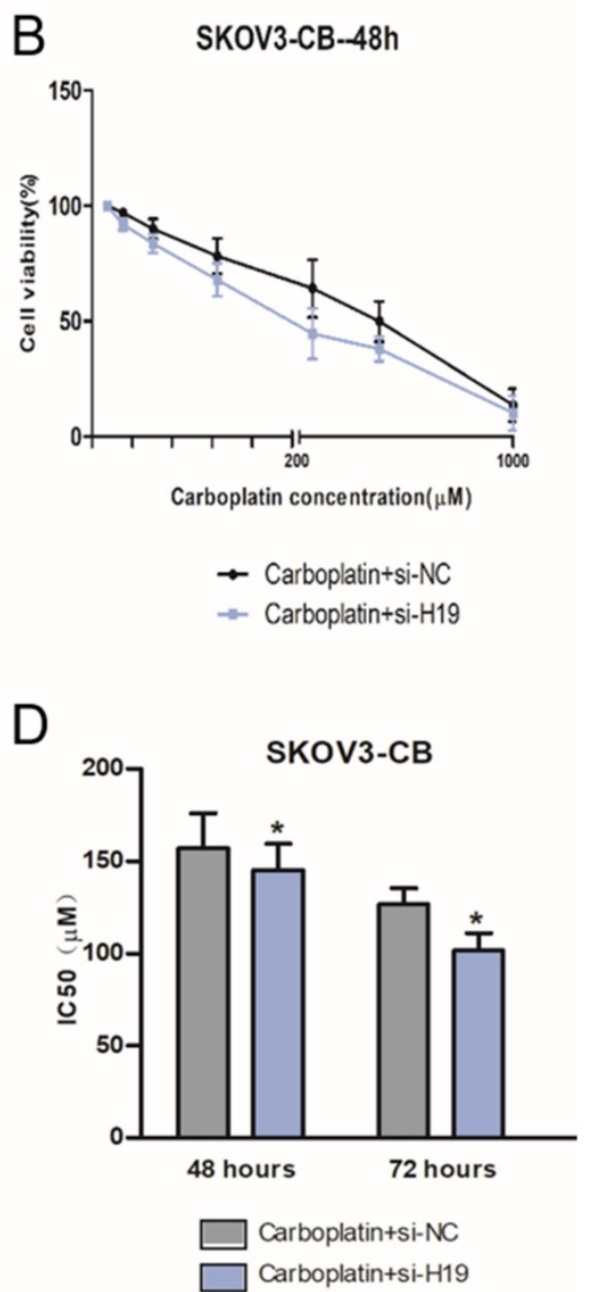

Figure 2: IncRNA-H19 strengthened ovarian cancer cell resistance to carboplatin. A-D) SKOV and SKOV3-CB cells were transfected with si-IncRNA-HI9 or the negative control and treated with different concentrations of carboplatin for $48 \mathrm{~h}$. Cell viability was measured by CCK-8 assays; E-H) IC50 value for carboplatin in 2 types of EOC cells transfected with si-IncRNA-H19 or negative control and treated with different concentrations determined using CCK-8 assays. Data are shown as the means \pm SEM. * $\mathrm{p}<0.05$ versus the negative control. 
activity was found in the pmirGLO-H19-Mut+ miR-29b-3p mimic group and the pmirGLO-H19-Wt+ miR-NC group (Fig. 4B). Furthermore, RT-qPCR experiments showed that a change in the miR-29b-3p level did not change lncRNA-H19 expression (Fig. 4C). However, higher lncRNA-H19 levels dramatically decreased miR-29b-3p levels in EOC cell lines $(\mathrm{P}<0.05)$ (Fig. 4D). These results indicated that lncRNA-H19 is an upstream regulator of miR-29b-3p. Ago2-RIP was used to validate the direct interaction of lncRNA-H19 and miR-29b-3p, which indicated that the Ago2-tagged wild-type IncRNA-H19 was observably enriched for miR-29b-3p compared to the empty vector and lncRNA-H19 with a mutated binding site (Fig. 4E). Further, the results of the RNA pulldown assay showed that lncRNA-H19 was pulled down by biotin-labeled miR-29b-3p oligos (Fig. 4F). Together, these results indicated that IncRNA-H19 served as a sponging lncRNA for miR-29b-3p.
A

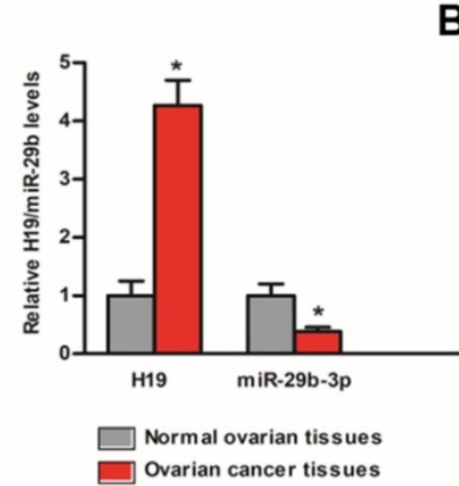

B

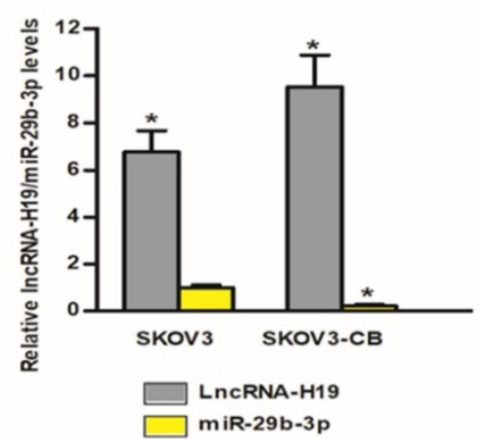

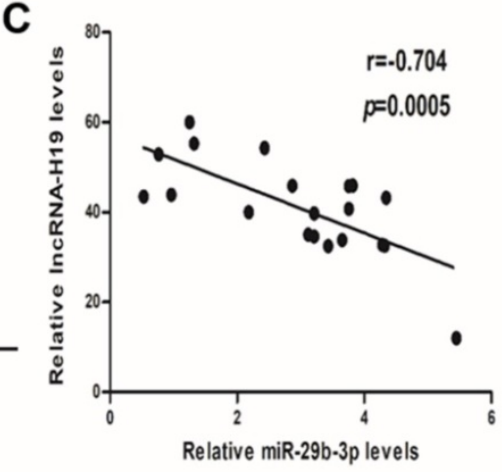

Figure 3: IncRNA-H19 and miR-29b-3p levels between EOC and control tissues/cells. A) IncRNA-H19 and miR-29b-3p levels were compared between EOC tissues and normal ovarian tissues. * P $<0.05$ vs. normal ovarian tissues; B) IncRNA-H1 9 and miR-29b-3p expression were compared in SKOV3 and SKOV3-CB cell lines. * IncRNA-H19 level $\mathrm{P}<0.05$ vs. SKOV3 cell line, \# miR-29b-3p level $\mathrm{P}<0.05$ vs. SKOV3 cell line; C) IncRNA-H19 and miR-29b-3p levels in EOC tissues were negatively correlated.

A $\begin{array}{ll}\text { LncH19 WT 3' } & \text { 5'- ccccatccacagAAATGGTGCTa -3' } \\ \text { miR-29b-3p } & \text { 3'- ttgtgactaag TIACCACGAC -5' } \\ \text { LncH19 Mut 3' } & \text { 5'-cccatccacagCATCAATTAGa -3' }\end{array}$

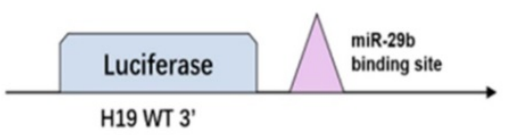

$\underset{\text { Luciferase }}{\stackrel{\text { H19 Mut 3' }}{\longrightarrow}}$

C

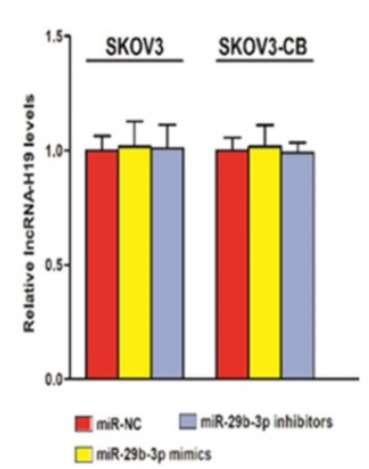

$\mathrm{D}$

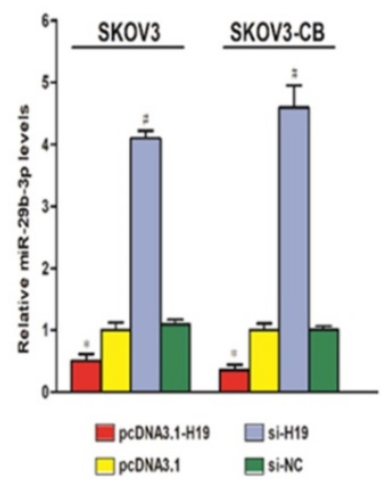

SKOV3

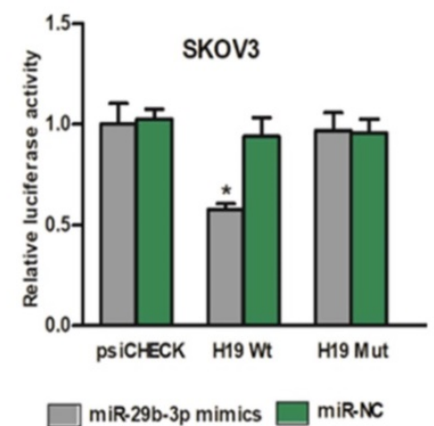

$\mathrm{E}$

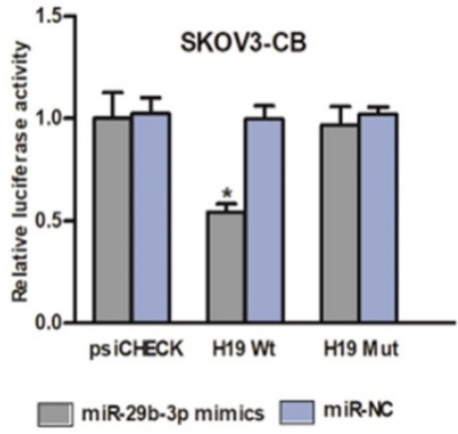

$\mathrm{F}$
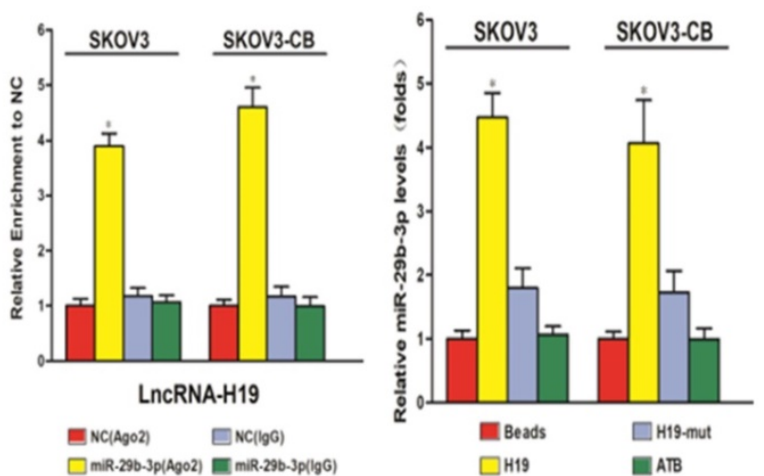

Figure 4: IncRNA-H19 sponged miR-29b-3p in EOC cells. A) IncRNA-H19 is targeted by miR-29b-3p; B-C) Luciferase activities in the SKOV3 and SKOV3-CB cell lines were compared between different transfection groups. * P $<0.05$ vs. the miR-29b-3p mimic+H19-Mut group; D) Influence of miR-29b-3p on IncRNA-H19 expression was determined in the SKOV3 and SKOV3-CB cell lines; E) Influence of IncRNA-H19 on miR-29b-3p expression was determined in the SKOV3 and SKOV3-CB cell lines; F) Ago2-RIP followed by miRNA RT-qPCR to detect the endogenous miR-29b-3p association with the Ago2-tagged IncRNA-H19 transcript; G) RNA pull-down assays were performed in EOC cells. IncRNA-H19 levels were measured by RT-qPCR. * P<0.05 vs. the negative control. miR/miRNA, microRNA; si, short interfering RNA; NC, negative control; ncRNA, noncoding RNA; IncRNA, long noncoding RNA; RIP, RNA binding protein immunoprecipitation. 

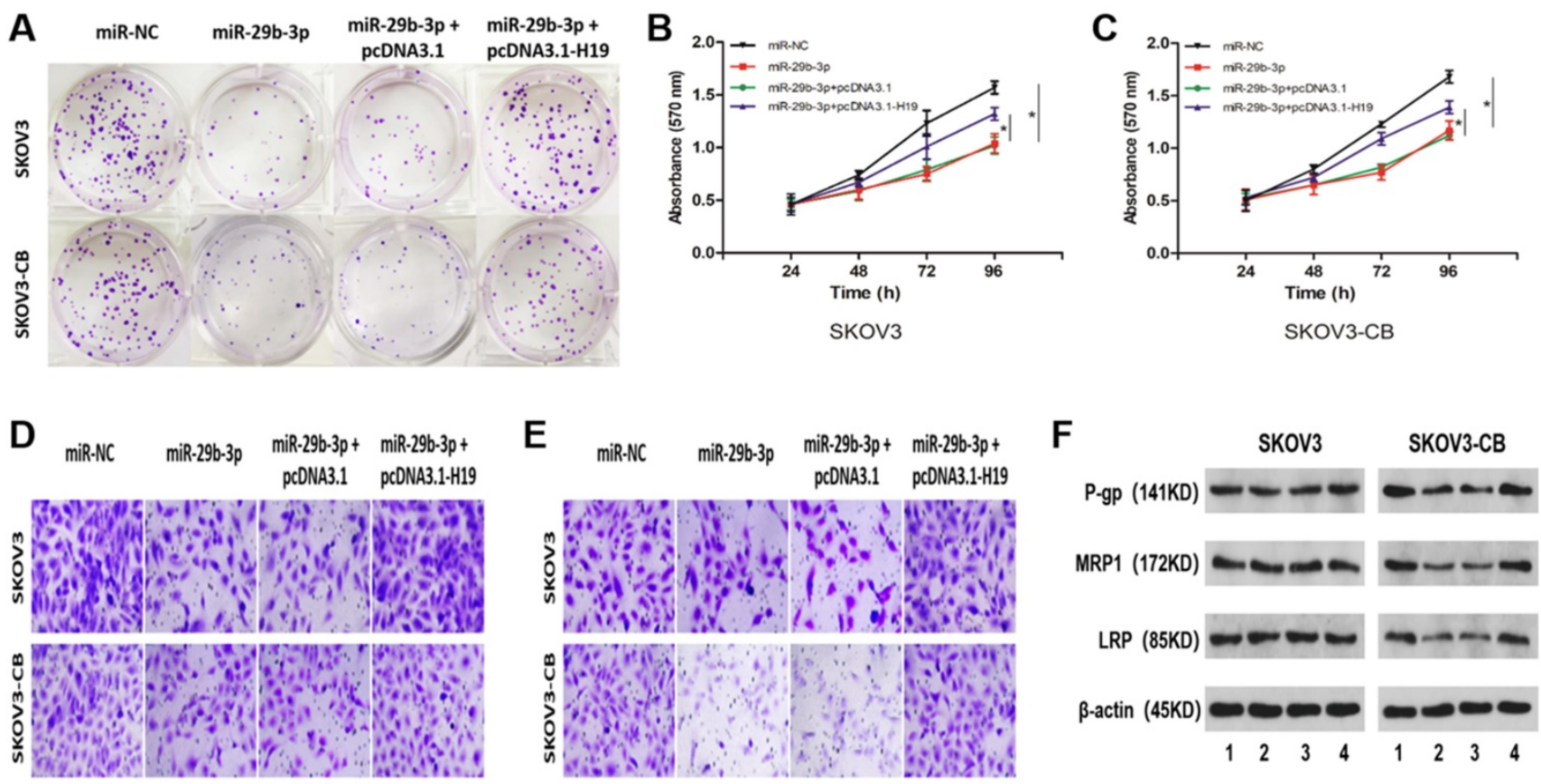

Figure 5: IncRNA-H19 attenuated miR-29b-3p-mediated carboplatin sensitivity in EOC cells. SKOV3 and SKOV3-CB cells were transfected with miR-NC, miR-29b-3p, miR-29b-3p+pcDNA3.1, or miR-29b-3p+pcDNA3.1-H19, followed by detection of colony number (A, 200x), cell proliferation (B and C), migration (D, 200x), invasion (E, 200x), and the drug-resistance-related proteins $\mathrm{P}$-gP, MRPI, and LRP (F and G). $* \mathrm{P}<0.05$. IC50, 50\% inhibitory concentration. For Figure $5 \mathrm{~F}$, lane $1-4$ stands for miR-NC, miR-29b-3p, miR-29b-3p + pcDNA3.1, and miR-29b-3p + pcDNA3.1-H19, respectively.

\section{LncRNA H19 attenuated miR-29b-3p-mediated carboplatin sensitivity in carboplatin-resistant EOC cells}

To further determine whether the lncRNA-H19/ miR-29b-3p axis affects chemosensitivity in EOC cells, we carried out rescue experiments by transfecting miR-NC, miR-29b-3p, miR-29b-3p+pcDNA3.1, or miR29b-3p+pcDNA3.1-H19 into SKOV3/SKOV3-CB cells. Overexpression of miR-29b-3p inhibited colony formation (Fig. 5A), cell proliferation (Fig. 5B and 5C), migration (Fig. 5D), and invasion (Fig. 5E). Interestingly, no significant difference was seen in the abovementioned cellular phenotypes between the two cell lines. However, western blot results indicated that exogenous expression of miR-29b-3p downregulated the drug resistance-associated proteins P-gp, MRP1, and LRP (Fig. 5F) in carboplatin-resistant SKOV3-CB cells. Moreover, restoration of H19 blocked all of the three miR-29b-3p-manipulated protein levels in carboplatin-resistant EOC cells (Fig. 5F). Collectively, these findings showed that IncRNA-H19 attenuated reactivity to carboplatin by blocking miR-29b-3p in carboplatin-resistant EOC cells.

\section{STAT3 was a direct target of miR-29b-3p and was negatively regulated by $\mathrm{miR}-29 \mathrm{~b}-3 \mathrm{p}$ in EOC cell lines}

We used miRanda and TargetScan software to predict the downstream targets of miR-29b-3p associated with EOC chemoresistance. STAT3 was identified as a potential target of miR-29b-3p considering its role in chemoresistance. As shown in Fig. 6A and 6B, miR-29b-3p mimic and inhibitor transfection caused decreased and increased STAT3 expression, respectively, at both the mRNA and protein levels. Therefore, STAT3 was negatively regulated by miR-29b-3p in EOC cell lines. miR-29b-3p showed complementarity with the STAT3 3'UTR sequence (Fig. 6C). This observation suggested that STAT3 might be inhibited by miR-29b-3p through complementary binding to its 3'UTR sequences. Finally, a 3'UTR luciferase reporter assay confirmed the direct binding between miR-29b-3p and the STAT3 3'UTR. Decreased luciferase expression was only observed in the cells transfected with the wild-type STAT3 3'UTR, and this suppressive effect of miR-29b-3p was blocked by miR-29b-3p binding site mutations in the STAT3 3'UTR (Fig. 6D). Collectively, these data suggested that miR-29b-3p negatively regulated STAT3 expression by complementary binding.

\section{The IncRNA H 19-miR-29b-3p-STAT3 axis regulated carboplatin sensitivity in EOC cells}

Rescue experiments were carried out in SKOV3/SKOV3-CB cells to explore the correlation between miR-29b-3p and STAT3 in the drug sensitivity of EOC cells. STAT3 knockdown increased the sensitivity of EOC cells to carboplatin, as indicated by the lower IC50 values (Fig. 7A) and colony 
formation (Fig. 7B), inhibited cell proliferation (Fig. $7 \mathrm{C}$ and 7D), migration (Fig. 7E), and invasion (Fig. 7F), and lower levels of drug-resistance-associated proteins P-gp, MRP1, and LRP (Fig. 7G and 7H) in both cell lines. However, inhibition of miR-29b-3p induced si-STAT3-mediated drug sensitivity in EOC cells (Fig. 5). To determine whether lncRNA-H19 regulated carboplatin resistance via the miR-29b-3p/ STAT3 axis, we transfected si- lncRNA-H19 into EOC cells together with control inhibitors or miR-29b-3p inhibitors. As shown in Fig. 7I, knockout of lncRNA-H19 significantly decreased STAT3 expression, which was restored by miR-29b-3p inhibition. Collectively, our findings indicated that the lncRNA-H19/miR-29b-3p/STAT3 axis is a regulatory pathway in the carboplatin resistance of EOC cells.

\section{Discussion}

Carboplatin is a widely used chemotherapeutic drug for EOC, but the development of carboplatin resistance substantially limits the treatment effect of chemotherapy. A growing number of studies have proven that IncRNA dysregulation is a possible reason for chemoresistance in different human cancers, including EOC [15] [16]. Identification of novel EOC-specific chemoresistance-associated lncRNAs is important for the future identification of
lncRNA-targeted treatment. Here, we found that lncRNA-H19 was highly expressed in carboplatinresistant EOC tumor tissues and cells. Admittedly, the sample size and control size in the current study are relatively small, and future expansion of sample size is in need to illustrate the study strength. Further, we proposed for the first time that lncRNA-H19 knockdown improved the sensitivity of EOC cells to carboplatin by suppressing STAT3 by functioning as a ceRNA of miR-29b-3p.

Currently, lncRNA-H19 was observed to regulate cell survival, migration, invasion and apoptosis by regulating target microRNAs [17] [18]. Various functional mechanisms have been identified between lncRNAs and miRNAs in tumor biology. The most studied functional mechanism for lncRNAs is acting as ceRNAs to block the binding of specific miRNAs and their target genes, which contributes to target mRNA derepression. In our study, we aimed to explore the functional mechanism of lncRNA-H19 in the carboplatin resistance of EOC. As a result, miR-29b-3p was observed to be a lncRNA-H19 target. Rescue experiments further demonstrated that miR-29b-3p sensitized EOC cells to carboplatin, and the effect of miR-29b-3p was reversed by lncRNA-H19 overexpression. Considered a versatile player in cancer development and progression, miR-29b-3p has been found to play a central role in the cancer
A

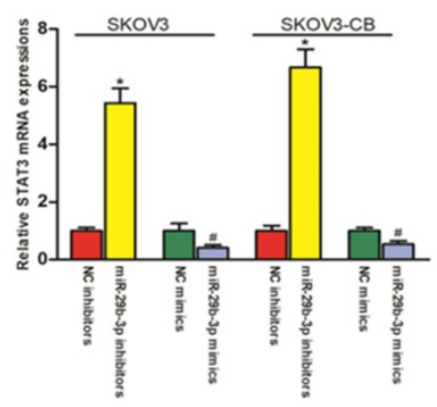

B

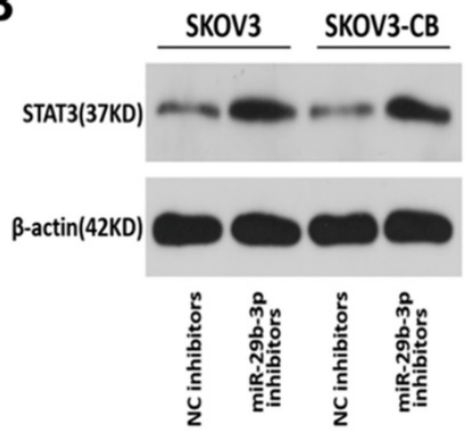

C STAT3 WT 3'UTR $\operatorname{miR}-29 b-3 p$ STAT3 Mut 3'UTR 5'- aguuaacagccuccuUGGUGCUu $-3^{\prime}$ I | III| 3'- uugugacuaaaguuuACCACGAu -5' (1)

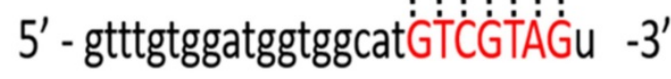

\section{Binding position: 1011-1017 of STAT3 3'UTR}

Figure 6: STAT3 was downregulated in EOC cells and was a downstream target of miR-29b-3p. qPCR (A) and Western blot (B) results show that miR-29b-3p mimics and inhibitors lowered and increased STAT3 expression, respectively, at both the mRNA and protein levels. C) Schematic of the STAT3 3'UTR luciferase reporter system. D) Luciferase reporter assay results confirmed the direct binding between miR-29b-3p and the STAT3 3 'UTR. Data are presented as the mean \pm SEM. * P $<0.05$ versus the control. 
A

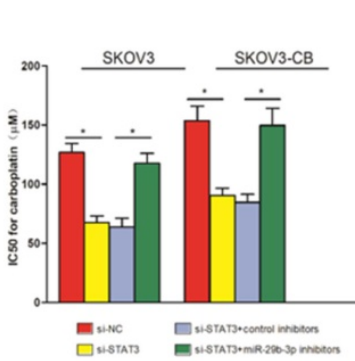

E

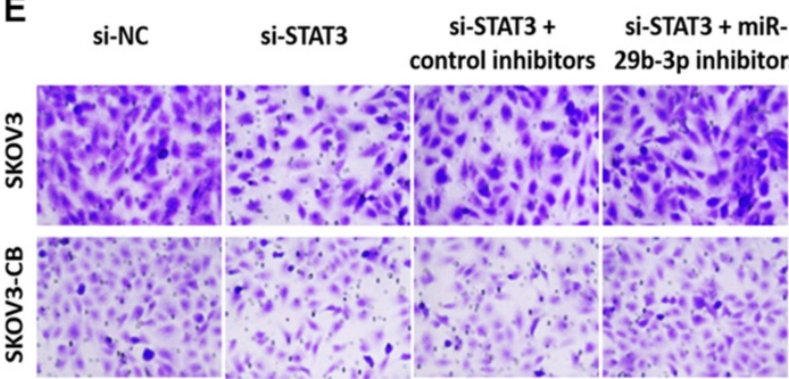

F

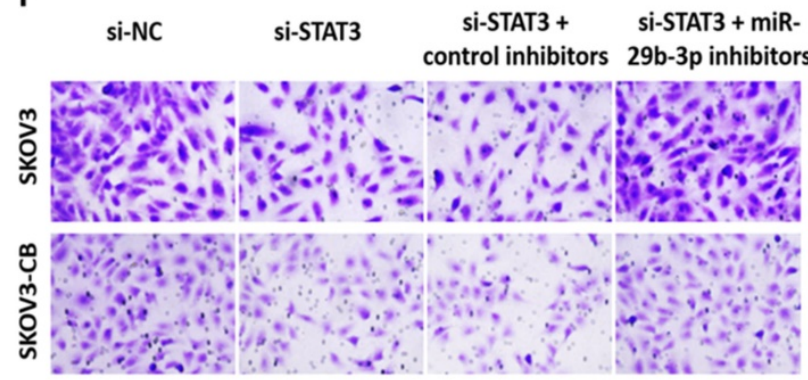

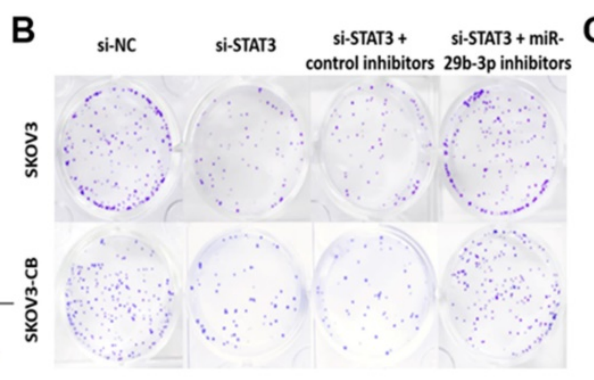
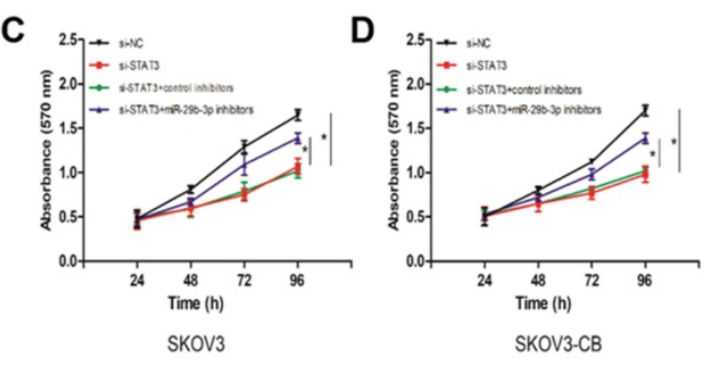

G

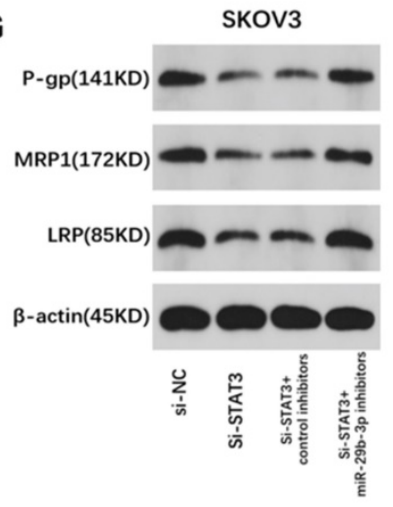

H

H SKOV3-CB

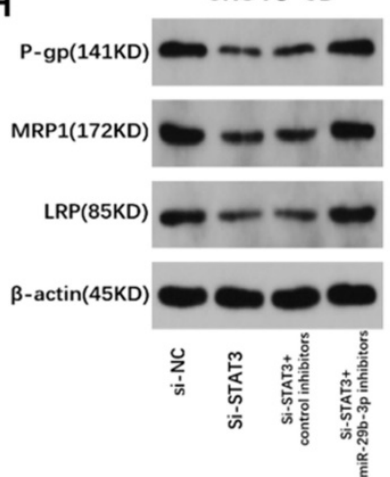

I

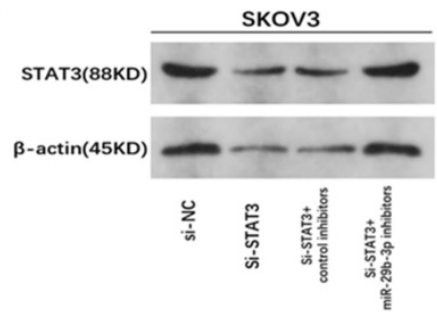

Figure 7: Role of the IncRNA-H19-miR-29b-3p-STAT3 axis in the carboplatin sensitivity of EOC cells.EOC cells were transfected with si-NC, si-STAT3, si-STAT3+anti-miR-NC, or si-STAT3+anti-miR-29b-3p, and then, the IC50 value for carboplatin was measured in each group (A), along with colony number (B, 200x), cell proliferation (C and D), migration (E, 200x), invasion (F, 200×), and P-gP, MRPI, and LRP proteins (G and H); I) STAT3 expression in SKOV3 and SKOV3-CB cells following the transfection of si-NC, si-STAT3, si-STAT3+control inhibitors, or si-STAT3+miR-29b-3p inhibitors. $*$ P $<0.05$.

response to chemotherapy in different cancer types [19] [20] [21]. However, no study has shown whether miR-29b-3p participates in the regulation of chemotherapy in EOC. Our study is the first to reveal the function and underlying mechanism of miR-29b-3p in enhancing carboplatin sensitivity in carboplatin-resistant EOC cells.

miRNAs are reported to negatively regulate target genes through binding with their 3'UTRs. In the current study, we showed that miR-29b-3p negatively regulates signal transducer and activator of transcription 3 (STAT3) by directly targeting their 3'UTR sequences, enriching our understanding of miR-29b-3p as a chemoresistance regulator in EOC development. STAT3 is a well-known oncogenic transcription factor and is increasingly associated with cancer initiation, progression, metastasis, chemoresistance, and immune evasion [22] [23]. STAT3 signaling has been found to negatively regulate the anticancer activity of chemotherapies in different cancers, including EOC [24] [25] [26]. Here, we found that knockdown of STAT3 enhanced the chemosensitivity of EOC cells to carboplatin, and the biological function of STAT3 was rescued by miR-29b-3p inhibitors. Moreover, lncRNA-H19 is a miR-29b-3p sponge, and lncRNA-H19 silencing repressed the miR-29b-3p downstream target STAT3. In addition, a recently published study observed that lncRNA-H19/miR-29b-3p/STAT3 signaling contributed to lung cancer progression by mediating cell viability, survival, apoptosis and the EMT process [14]. Our and other findings indicated that the specific role and underlying molecular mechanism of the lncRNA-H19/miR-29b-3p/STAT3 pathway in the initiation and development of cancer should be further explored in the future.

\section{Conclusion}

This study revealed that lncRNA-H19 and STAT3 were significantly increased but miR-29b-3p was decreased in carboplatin-resistant EOC. Silencing lncRNA-H19 improved carboplatin effectiveness in 
EOC cells. LncRNA-H19 is a competing endogenous RNA of miR-29b-3p that further regulates the miR-29b-3p downstream target STAT3, and STAT3 eventually leads to resistance to carboplatin in EOC cells. In conclusion, we propose that the lncRNA-H19/miR-29b-3p axis enhances carboplatin resistance in EOC by targeting STAT3 and may provide a possible way to improve chemotherapy for EOC.

\section{Supplementary Material}

Supplementary figure.

http://www.jcancer.org/v12p5712s1.pdf

\section{Acknowledgements}

The authors gratefully thank the Natural Science Basic Research Plan in Shaanxi Province of China for funding this research.

\section{Ethics approval and consent to participate}

Human ovarian carcinomas and matched normal ovarian tissue samples were collected from patients at The First Affiliated Hospital of $\mathrm{Xi}^{\prime}$ an Jiaotong University, PR China. This study was approved by the Ethics Committee of The First Affiliated Hospital of Xi'an Jiaotong University, PR China. Written consent was obtained from each study participant enrolled.

\section{Availability of data and materials}

All data were analyzed by SPSS 22.0 software and presented as mean $\pm \mathrm{SD}$.

\section{Funding}

This work was supported by the Natural Science Basic Research Plan in Shaanxi Province of China (No. 2018JM7014).

\section{Author Contributions}

Study design: YT, XYT, XHZ; literature research and cell culture: $X Y T, X H Z$; experimental studies: XYT, XHZ, CL; data analysis: CL, MH; manuscript preparation: $\mathrm{XYT}, \mathrm{XHZ}$; manuscript editing: $\mathrm{MH}$; manuscript review: CL, YT. All authors read and approved the final manuscript.

\section{Competing Interests}

The authors have declared that no competing interest exists.

\section{References}

1. Marchetti C, Pisano C, Facchini G, et al. First-line treatment of advanced ovarian cancer: current research and perspectives. Expert review of anticancer therapy. 2010; 10(1): 47-60.

2. Gayen S, Maclary E, Buttigieg E, et al. A Primary Role for the Tsix lncRNA in Maintaining Random X-Chromosome Inactivation. Cell reports. 2015; 11(8): 1251-1265.
3. Kawaguchi T, Hirose T. Chromatin remodeling complexes in the assembly of long noncoding RNA-dependent nuclear bodies. Nucleus (Austin, Tex). 2015; 6(6): 462-467.

4. Zhuang W, Ge X, Yang S, et al. Upregulation of lncRNA MEG3 Promotes Osteogenic Differentiation of Mesenchymal Stem Cells From Multiple Myeloma Patients By Targeting BMP4 Transcription. Stem cells (Dayton, Ohio). 2015; 33(6): 1985-1997.

5. Inamura K. Major Tumor Suppressor and Oncogenic Non-Coding RNAs: Clinical Relevance in Lung Cancer. Cells. 2017; 6(2): 12.

6. Shukla GC, Singh J, Barik S. MicroRNAs: Processing, Maturation, Target Recognition and Regulatory Functions. Molecular and cellular pharmacology. 2011; 3(3): 83-92.

7. Zheng X, Zhou Y, Chen W, et al. Ginsenoside 20(S)-Rg3 Prevents PKM2-Targeting miR-324-5p from H19 Sponging to Antagonize the Warburg Effect in Ovarian Cancer Cells. Cellular physiology and biochemistry : international journal of experimental cellular physiology, biochemistry, and pharmacology. 2018; 51(3): 1340-1353.

8. Li J, Huang $\mathrm{Y}$, Deng X, et al. Long noncoding RNA H19 promotes transforming growth factor-beta-induced epithelial-mesenchymal transition by acting as a competing endogenous RNA of miR-370-3p in ovarian cancer cells. OncoTargets and therapy. 2018; 11: 427-440.

9. Chen Z, Wei H, Zhao X, et al. Metformin treatment alleviates polycystic ovary syndrome by decreasing the expression of MMP-2 and MMP-9 via H19/miR-29b-3p and AKT/mTOR/autophagy signaling pathways. J Cell Physiol. 2019; 234(11): 19964-19976.

10. Teng Y, Zuo X, Hou M, et al. A Double-Negative Feedback Interaction between MicroRNA-29b and DNMT3A/3B Contributes to Ovarian Cancer Progression. Cellular physiology and biochemistry : international journal of experimental cellular physiology, biochemistry, and pharmacology. 2016; 39(6): 2341-2352.

11. Teng Y, Zhang Y, Qu K, et al. MicroRNA-29B (mir-29b) regulates the Warburg effect in ovarian cancer by targeting AKT2 and AKT3. Oncotarget. 2015; 6(38): 40799-40814.

12. Teng $Y$, Zhao L, Zhang $Y$, et al. Id-1, a protein repressed by miR-29b, facilitates the TGFbeta1-induced epithelial-mesenchymal transition in human ovarian cancer cells. Cellular physiology and biochemistry : international journal of experimental cellular physiology, biochemistry, and pharmacology. 2014; 33(3): 717-730.

13. Luan $\mathrm{YZ}, \mathrm{Li} \mathrm{L}, \mathrm{Li} \mathrm{DR}$, et al. [Establishment of 5 resistant ovarian cancer cell strains and expression of resistance-related genes]. Zhonghua fu chan ke za zhi. 2004; 39(6): 403-407.

14. Liu L, Liu L, Lu S. IncRNA H19 promotes viability and epithelial-mesenchymal transition of lung adenocarcinoma cells by targeting miR-29b-3p and modifying STAT3. International journal of oncology. 2019; 54(3): 929-941.

15. Zhao L, Yang J, Liu X, et al. Overexpression of Long Noncoding RNA E2F-Mediated Cell Proliferation Enhancing Long Noncoding RNA Is Involved in the Development of Chemoresistance of Cancer Cells to Carboplatin in Ovarian Endometrioid Adenocarcinoma. Cancer biotherapy \& radiopharmaceuticals. 2019; 34(9): 566-671.

16. Qu C, Dai C, Guo Y, et al. Long noncoding RNA SNHG15 serves as an oncogene and predicts poor prognosis in epithelial ovarian cancer. OncoTargets and therapy. 2019; 12: 101-111.

17. Zhou Y, Fan RG, Oin CL, et al. LncRNA-H19 activates CDC42/PAK1 pathway to promote cell proliferation, migration and invasion by targeting miR-15b in hepatocellular carcinoma. Genomics. 2018; 111(6): 1862-1872.

18. Ghaedi H, Mozaffari MAN, Salehi Z, et al. Co-expression profiling of plasma miRNAs and long noncoding RNAs in gastric cancer patients. Gene. 2019; 687: 135-142.

19. Luo DJ, Li LJ, Huo HF, et al. MicroRNA-29b sensitizes osteosarcoma cells to doxorubicin by targeting matrix metalloproteinase 9 (MMP-9) in osteosarcoma. European review for medical and pharmacological sciences. 2019; 23(4): 1434-1442.

20. Pan Y, Zhang Y, Liu W, et al. LncRNA H19 overexpression induces bortezomib resistance in multiple myeloma by targeting MCL-1 via miR-29b-3p. Cell death \& disease. 2019; 10(2): 106.

21. $\mathrm{Xu} \mathrm{W}, \mathrm{Li} \mathrm{Z}, \mathrm{Zhu} \mathrm{X}$, et al. miR-29 Family Inhibits Resistance to Methotrexate and Promotes Cell Apoptosis by Targeting COL3A1 and MCL1 in Osteosarcoma. Medical science monitor : international medical journal of experimental and clinical research. 2018; 24: 8812-8821.

22. Huynh J, Chand A, Gough D, et al. Therapeutically exploiting STAT3 activity in cancer - using tissue repair as a road map. Nature reviews Cancer. 2019; 19(2): 82-96.

23. Johnson DE, O'Keefe RA, Grandis JR. Targeting the IL-6/JAK/STAT3 signalling axis in cancer. Nature reviews Clinical oncology. 2018; 15(4): 234-248. 
24. Furtek SL, Backos DS, Matheson CJ, et al. Strategies and Approaches of Targeting STAT3 for Cancer Treatment. ACS chemical biology. 2016; 11(2): 308-318.

25. Tzeng YT, Liu PF, Li JY, et al. Kinome-Wide siRNA Screening Identifies Src-Enhanced Resistance of Chemotherapeutic Drugs in Triple-Negative Breast Cancer Cells. Frontiers in pharmacology. 2018; 9: 1285.

26. Park GB, Kim D. MicroRNA-503-5p Inhibits the CD97-Mediated JAK2/STAT3 Pathway in Metastatic or Paclitaxel-Resistant Ovarian Cancer Cells. Neoplasia (New York, NY). 2019; 21(2): 206-215. 